\title{
Outcomes of Proton Beam Radiotherapy for Large Non-Peripapillary Choroidal and Ciliary Body Melanoma at TRIUMF and the BC Cancer Agency
}

\author{
Britta Weber ${ }^{a, c}$ Katherine Paton ${ }^{b}$ Roy Ma ${ }^{a}$ Tom Pickles ${ }^{a}$ \\ ${ }^{a}$ Radiation Oncology, British Columbia Cancer Agency and University of British Columbia, and bepartment of \\ Ophthalmology and Visual Sciences, Vancouver Hospital Eye Care Center and University of British Columbia, \\ Vancouver, B.C., Canada; ' Department of Oncology, Aarhus University Hospital, Aarhus, Denmark
}

\section{Key Words}

Proton therapy · Uveal melanoma · Ciliary body · Outcomes · Toxicity

\begin{abstract}
Background and Purpose: To report outcomes and toxicity after proton beam radiotherapy for non-peripapillary choroidal and ciliary body melanoma considered unsuitable for other eye-sparing therapies. Materials and Methods: An existing database of 77 patients with non-peripapillary tumors treated at TRIUMF, Canada, including patient, tumor, and treatment characteristics, was updated with ocular complications and follow-up status from chart reviews. Results: Most of the patients had large tumors: $61 \%$ were T3/T4 tumors (AJCC classification), while $48 \%$ were large by the Collaborative Ocular Melanoma Study classification. The median thickness was $7.1 \mathrm{~mm}$, and the ciliary body was involved in $35 \%$. After 5 and 10 years, the actuarial ocular tumor control rate was 85 and $85 \%$, metastasis-free survival was 72 and $57 \%$, overall survival was 77 and $63 \%$, the enucleation rate was 22 and $22 \%$, and complete blindness was found in 38 and $38 \%$, respectively. On univariate analysis, patients with ciliary body involvement had significantly worse metastasisfree survival and overall survival rates compared to patients without ciliary body involvement $(p<0.001)$. Conclusions:
\end{abstract}

Proton therapy for large anteriorly located tumors resulted in acceptable ocular tumor control and survival rates. The risk of blindness and severe toxicity requiring enucleation was low, and a substantial proportion of patients maintained useful vision.

(c) 2015 S. Karger AG, Basel

\section{Introduction}

Ocular melanoma is an uncommon malignancy affecting mostly Caucasians. The incidence rate is highest in Northern Europe, where it reaches more than 8 per million each year [1]. Enucleation of the affected eye was the standard treatment of choroidal melanoma for most of the last century, but since the 1960s/1970s brachytherapy has been commonly used in small and medium-sized tumors suitable for the technique. In 2002, the results of the randomized Collaborative Ocular Melanoma Study (COMS) demonstrated equal efficacy of episcleral plaque brachytherapy compared to enucleation [2]. At the same time, other eye-sparing radiation techniques such as proton therapy and stereotactic radiotherapy were also established [2-7]. The advantage of these new treatment options is that the eye is preserved, that useful vision is maintained in some patients, and that there is excellent

\section{KARGER}

E-Mail karger@karger.com

www.karger.com/oop (c) 2015 S. Karger AG, Base

2296-4681/15/0021-0029\$39.50/0
Prof. Tom Pickles, MD

Department of Radiotherapy and Developmental Radiotherapeutics University of British Columbia, Radiation Oncology, BC Cancer Agency 600 West 10th Ave, Vancouver, BC V6R 2T9 (Canada)

E-Mail tpickles@bccancer.bc.ca 
tumor control and survival in most patients. However, vision loss might be inevitable due to radiation side effects such as radiation retinopathy or intractable neovascular glaucoma (NVG). The size of the tumor, together with the location of the tumor, must be taken into account when the optimal treatment is chosen. Proton therapy is feasible in a larger range of tumors compared to brachytherapy and stereotactic radiotherapy, as both posterior peripapillary tumors as well as anteriorly located tumors can be treated $[2-4,7,8]$. This is particularly in larger tumors and tumors with extrascleral extension where brachytherapy is not recommended $[5,9,10]$. The physical properties of the proton beam, with a low entrance dose, followed by a very high dose to the target area (determined by the Bragg peak), and a subsequent rapid fall-off of the dose to zero distal to the target makes this treatment attractive in organs with small distances to critical structures such as the eye.

Since 1995, the BC Cancer Agency, in conjunction with the University of British Columbia Eye Care Centre and TRIUMF (Tri-University Meson Facility), has been operating the only proton therapy facility in Canada for the treatment of patients with ocular melanomas. Indications for proton therapy include large tumors, tumors with extension into the ciliary body or extrascleral extension, and peripapillary tumors unsuitable for brachytherapy.

We have previously reported our results of proton therapy in posterior peripapillary tumors [11]. This is the first report of the outcome and toxicity of patients with non-peripapillary tumors treated with protons from 1995 to 2013 at TRIUMF in Canada.

\section{Materials and Methods}

We conducted a retrospective study of all patients with nonperipapillary melanoma, defined as tumors located more than $2 \mathrm{~mm}$ from the optic disc, and treated with proton therapy between 1995 and 2013. Patients with iris melanoma were not included. Patients were referred to ocular oncology services from across Canada, but mainly from ophthalmologists from Western provinces. All patients were seen and examined by a dedicated ophthalmologist specialized in ocular oncology (K.P.). The examinations included slit-lamp examination, visual acuity, intraocular pressure measurements, direct and indirect ophthalmoscopy, fundus photography, fluorescein angiography, optical coherence tomography, once available, and A- and B-mode ultrasound for tumor measurements. Staging investigations included chest radiography, liver enzyme levels, and liver ultrasound to exclude distant metastases. To confirm eligibility, the patients were also assessed by one of two radiation oncologists (T.P. or R.M.) at the BC Cancer Agency.

Baseline patient and tumor characteristics as well as proton treatment details and dosimetric parameters were prospectively
Table 1. Patient and tumor characteristics of 77 patients with choroidal and ciliary body melanoma treated with proton therapy from 1995 to 2013

\begin{tabular}{|c|c|}
\hline Age, years & $60(28-88)$ \\
\hline \multicolumn{2}{|l|}{ Gender } \\
\hline Female & $31(40)$ \\
\hline Male & $46(60)$ \\
\hline \multicolumn{2}{|l|}{ Affected eye } \\
\hline Left & $29(38)$ \\
\hline Right & $48(62)$ \\
\hline \multicolumn{2}{|l|}{ Location } \\
\hline Anterior & $41(53)$ \\
\hline Posterior & $36(47)$ \\
\hline \multicolumn{2}{|l|}{ Uveal melanoma } \\
\hline Choroidal & $68(88)$ \\
\hline Ciliary body & $9(12)$ \\
\hline \multicolumn{2}{|l|}{ Tumor characteristics } \\
\hline Thickness, mm & $7.1(1.4-12.6)$ \\
\hline Diameter, mm & $13.6(3.5-21.0)$ \\
\hline \multicolumn{2}{|l|}{ T stage } \\
\hline 1 & $9(12)$ \\
\hline 2 & $21(27)$ \\
\hline 3 & $43(56)$ \\
\hline 4 & $4(5)$ \\
\hline \multicolumn{2}{|l|}{ Stage } \\
\hline I (T1a) & $3(4)$ \\
\hline IIa (T1b-d, T2a) & $22(29)$ \\
\hline IIb (T2b, T3a) & $35(45)$ \\
\hline IIIa (T2c-d, T3b-c, T4a) & $14(18)$ \\
\hline IIIb $(\mathrm{T} 3 \mathrm{~d}, \mathrm{~T} 4 \mathrm{~b}-\mathrm{c})$ & $2(3)$ \\
\hline IIIc (T4d-e) & $1(1)$ \\
\hline \multicolumn{2}{|l|}{ Ciliary body involvement } \\
\hline No & $50(65)$ \\
\hline Yes $^{\mathrm{a}}$ & $27(35)$ \\
\hline \multicolumn{2}{|l|}{ Angle involvement } \\
\hline No & $65(85)$ \\
\hline Yes & $11(14)$ \\
\hline Unknown & $1(1)$ \\
\hline \multicolumn{2}{|l|}{ Dose } \\
\hline $54 \mathrm{~Gy}$ & $8(10)$ \\
\hline $60 \mathrm{~Gy}$ & $69(90)$ \\
\hline \multicolumn{2}{|l|}{ Baseline vision } \\
\hline$\geq 20 / 50$ & $50(65)$ \\
\hline$\geq 20 / 200$ & $67(87)$ \\
\hline
\end{tabular}

Values are presented as $\mathrm{n}(\%)$ or median (range).

${ }^{a}$ Nine patients had a ciliary body tumor, while 18 had a choroidal tumor growing into the ciliary body.

recorded in a database at the BC Cancer Agency. Tumor size was recorded initially ( $\mathrm{T}$ staging), but all patients were restaged retrospectively based on the 2010 American Joint Committee on Cancer (AJCC) TNM classification for uveal melanoma [12]. Based on the tumor size according to T staging, ciliary body invasion, and extrascleral extension, patients were classified into six anatomic stages: Ia, IIa, IIb, IIIa, IIIb, and IIIc (table 1).
Weber/Paton/Ma/Pickles 
Table 2. Outcomes (actuarial, Kaplan-Meier) after proton therapy according to the anatomic stage

\begin{tabular}{lccc}
\hline Outcome $^{\mathrm{a}}$ & 2 years & 5 years & 10 years \\
\hline Ocular control & & & \\
All patients & 98.5 & 85.1 & 85.1 \\
Stage 1 (Ia) & 100 & - & - \\
Stage 2 (IIa + IIb) & 94.3 & 89.2 & 89.2 \\
Stage 3 (IIIa + IIIb + IIIc) & 100 & 66.7 & 66.7 \\
Metastasis-free survival & & & \\
All patients & 89.6 & 71.6 & 57.2 \\
Stage 1 & 100 & - & - \\
Stage 2 & 93.8 & 81.8 & 69.2 \\
Stage 3 & 73.7 & 35.2 & 11.7 \\
Overall survival & & & \\
All patients & 91.1 & 76.8 & 62.7 \\
Stage 1 & 100 & - & - \\
Stage 2 & 95.9 & 86.3 & 74.0 \\
Stage 3 & 74.0 & 44.2 & 17.7 \\
\hline
\end{tabular}

The anatomic stages are defined by the AJCC 2010 classification of uveal melanoma. ${ }^{\text {a }}$ Actuarial rates.

After treatment, patients were followed up on a regular basis by the ophthalmologist with the purpose of recording ocular recurrence and toxicity, and initiating treatment promptly when required. Patients from other provinces in Canada underwent shared follow-up by the referring ophthalmologist and the ocular oncologist, and written information was sent to the ocular oncologist after each visit. For this report, toxicities, ocular recurrence, and survival were reviewed retrospectively from ophthalmologist charts at the Eye Care Center and the BC Cancer Agency Information System (CAIS) and updated into the database.

\section{Treatment Procedures}

We have previously described our treatment procedures in detail [11].

Briefly, the first step is to implant tantalum clips for planning purposes on the sclera. A three-dimensional reconstruction of the tumor is made based on the clips, and the tumor borders are delineated by the ocular oncologist and verified by the radiation oncologist. Treatment is planned by use of EYEPLAN software, developed originally by Goitein and Miller [13] for dosimetric calculations. A margin of $2.5 \mathrm{~mm}$ around the tumor edges is added to the planning target volume, and a brass collimator shaping the treatment beam to the planning target volume is custom-made for each patient. All patients in our study were treated with 4 fractions on 4 consecutive days at the TRIUMF cyclotron facility at the University of British Columbia. From 1995 to 1997, the prescription dose was 60 cobalt gray equivalent (CGE; corresponding to 50 Gy multiplied by the relative biological effectiveness of protons of 1.2 as measured on our beam line [14]). However, in 1997, the dose was reduced to 54 CGE due to a concerning high rate of secondary glaucoma. In 2001, the dose was changed back to 60 CGE because of a clinical concern of loss of tumor control [11]. The proton beam was given with energies of 70 (early years) to $74 \mathrm{MeV}$ (later years), which provided a depth coverage by the $90 \%$ isodose curve of $34.3 \mathrm{~mm}$.

Proton Therapy in Large Uveal Melanoma

\section{Statistical Analysis}

The actuarial method of Kaplan and Meier was used to estimate ocular recurrence, metastasis-free survival, and overall survival rates in the whole group, and according to $\mathrm{T}$ classification and anatomic stages defined by the 2010 AJCC uveal melanoma staging system [12]. For the purpose of graphical comprehension, the six anatomic stages are grouped in three: stage I versus stage II versus stage III. Cumulative incidences, at 2, 5 and 10 years, were calculated for the major radiation toxicities, including the rate of enucleation and vision loss.

Univariate Analysis

The $\chi^{2}$ test was used to compare various categorical variables, and the log-rank test was used for quantitative censored data. $p$ values $<0.05$ derived from two-sided tests were considered statistically significant. Statistical analyses were performed with SPSS software, version 21 .

\section{Results}

A total of 173 patients were treated with proton therapy from 1995 to 2013 . We excluded 72 patients with peripapillary tumors (already published by Tran et al. [11]), 15 patients with iris melanoma, 2 patients with conjunctival tumors, 4 patients with recurrent disease after brachytherapy, and 3 patients with hemangioma. Patient and tumor characteristics of the 77 patients that met the inclusion criteria are summarized in table 1 . The median age was 60 years, ranging from 28 to 88 years. The median tumor diameter was $13.6 \mathrm{~mm}$, and the median thickness (height) was $7.1 \mathrm{~mm}$. Most patients (53\%) had a tumor located anterior to the equator, and in 27 patients (35\%) the ciliary body was involved. Of these 27 patients, 9 patients had a tumor arising in the ciliary body, while 18 had a choroidal tumor with extension into the ciliary body. Concerning other relevant medical history, 36\% had hypertension and $8 \%$ diabetes. One patient had pretreatment glaucoma.

The median follow-up time was 47 months (0-221). One patient was lost to follow-up immediately after treatment as he lived out of the country, and 5 patients did not complete follow-up as they were from other provinces in Canada. The outcomes are summarized in table 2.

\section{Overall and Metastasis-Free Survival}

The overall survival rate was $77 \%$ at 5 years and $63 \%$ at 10 years. The cause of death was malignant melanoma in $80 \%$ of the cases. The 5 - and 10 -year metastasis-free survival rate was 72 and $57 \%$, respectively.

The median time from treatment to metastasis was 36 months (1-183), and the median time from metastasis to 


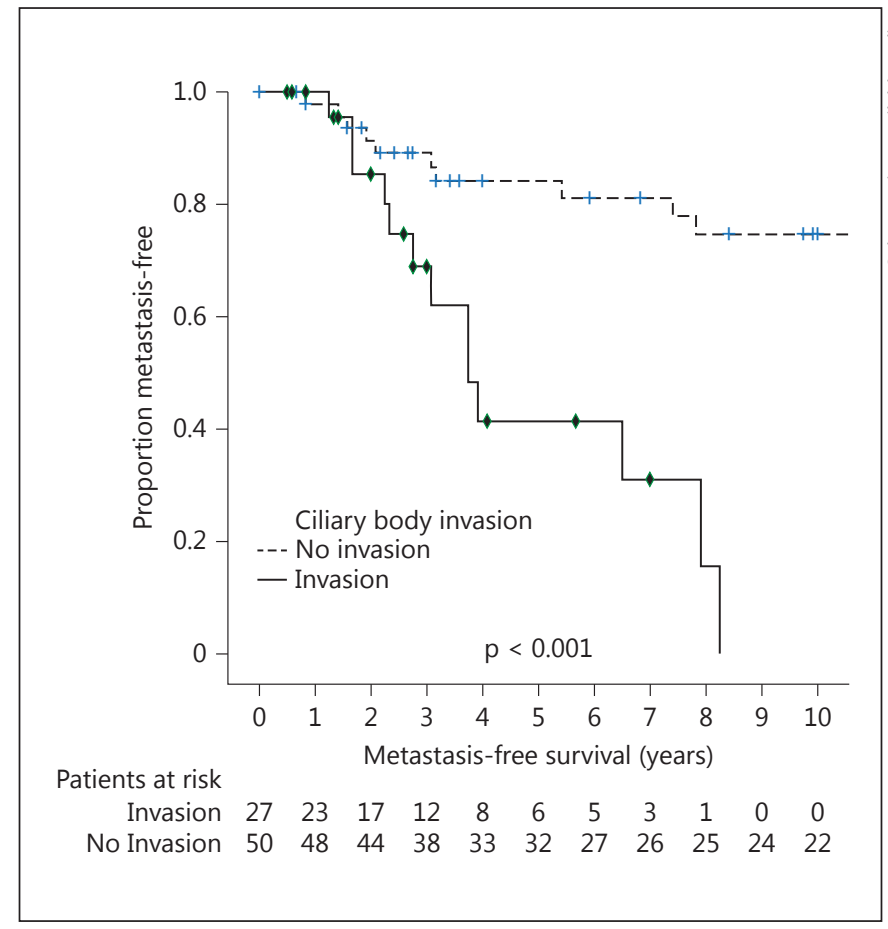

Fig. 1. Kaplan-Meier curve demonstrating metastasis-free survival according to ciliary body involvement.

death was 3 months $(0-52)$. In total, 26 patients developed metastasis; in 9 patients metastatic spread occurred more than 5 years after irradiation, and in 3 patients it occurred after more than 10 years.

On univariate analysis, four (not independent) factors were found to be associated with an increased rate of metastasis and death: anterior location, ciliary body involvement (fig. 1), high T stage (fig. 2a), and high anatomic stage according to the 2010 AJCC classification (fig. 2b). The difference found regarding $\mathrm{T}$ stage was only seen in patients with T4 tumors (fig. 2a), whereas there was no difference between T1, T2, and T3 tumors. In contrast, there was a well-defined separation of the patients according to anatomic stage (fig. 2b).

When tumors with invasion into the ciliary body were removed from the analysis, the anteriorly located tumors still had a significantly worse prognosis compared to the posteriorly located tumors $(\mathrm{p}=0.016)$. When comparing the tumor size in this group, we found significantly bigger dimensions of both the longest diameter and the height in the anteriorly located tumors compared to the posterior group ( 14.9 vs. $12.9 \mathrm{~mm}, \mathrm{p}=0.013$ and 8.6 vs. $6.4 \mathrm{~mm}$, $\mathrm{p}=0.001)$.

\section{Ocular Control}

The actuarial ocular recurrence rate at 5 years was $15 \%$ (7 patients), and no ocular recurrences were seen after 5 years. The median time to recurrence was 37 months (13-47). Only 1 recurrence was within the radiation field, whereas 6 were out-of-field recurrences. Four of these were in the choroid, but anatomically distant from the primary tumor, and 2 were extraocular recurrences on the sclera, also distant from the primary tumor site. Three patients had metastatic spread at the same time as ocular recurrence, and 3 had metastasis diagnosed within 2 years of ocular recurrence.

The management of ocular recurrence was enucleation in 2 cases, stereotactic radiation in 1 case, transpupillary thermotherapy in 1 patient, and no treatment in 3 cases ( 1 patient declined treatment, and 2 patients had rapidly progressing metastatic disease).

\section{Enucleation}

In total, 12 patients were enucleated. The 2 -year enucleation rate was $4.2 \%$, whereas the rate was $22 \%$ after both 5 and 10 years. Two patients were enucleated due to ocular recurrence as mentioned above, and 10 patients (87\%) due to toxicity. The median time to enucleation due to toxicity was 35 months (5-125). In 9 out of 10 patients, the reason was NVG causing a blind and painful eye. In 1 patient, an intravitreal hemorrhage caused high pressure and pain and led to enucleation.

\section{Visual Acuity}

Out of 77 patients, 75 had a pretreatment visual acuity of counting fingers or better. Conservation of such vision was achieved in $73.2 \%$ after 2 years and in $61.4 \%$ after 5 and 10 years.

Out of 77 patients, 67 had an initial visual acuity of $20 / 200$ or better. Conservation of such vision was achieved in $56.8 \%$ after 2 years and in $37 \%$ after 5 and 10 years.

Out of 77 patients, 50 had a good pretreatment vision of 20/50 or better in the tumor-affected eye. Conservation of this vision was observed in $60.4 \%$ after 2 years and in $39.5 \%$ after 5 and 10 years.

Tumor height was highly predictive for loss of vision on univariate analysis $(\mathrm{p}=0.001)$.

\section{Ocular Complications}

The main ocular complications were radiation retinopathy $(25 \%)$, cataract $(54 \%)$, rubeosis $(45 \%)$, and $\mathrm{NVG}$ (23\%). Medical treatment was initiated, including bevacizumab injections when rubeosis and/or NVG were diagnosed. In half of the 35 patients who developed rubeosis, 

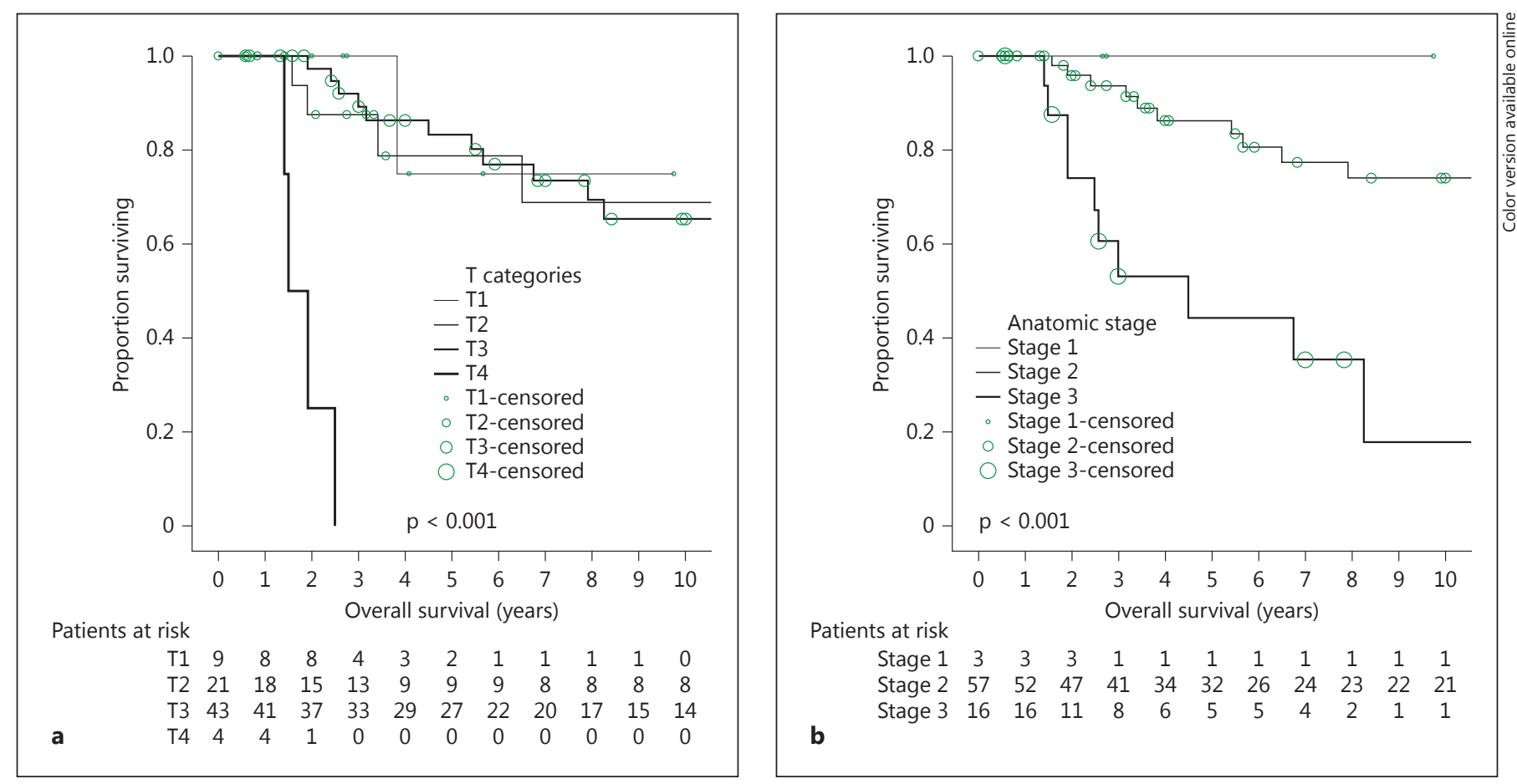

Fig. 2. Kaplan-Meier curves showing overall survival according to $T$ classification (a) and anatomic stage (b).

NVG was avoided, and $37 \%$ of these patients maintained a visual acuity of more than $20 / 200$, and $84 \%$ could still count fingers. In the other half (16 patients), where NVG could not be avoided, 9 patients ended up having enucleation. Patients with NVG who kept their eye had mostly no vision at all (83\%). In contrast, only $7 \%$ of patients without NVG ended up with no light perception $(\mathrm{p}<0.001)$.

The risk of developing NVG was very small in tumors with ciliary body invasion, probably due to a very anterior location and lower total volume of retina irradiated ( 4 vs. $34 \%, \mathrm{p}=0.003$ ).

On univariate analysis, the volume of retina getting more than 20 Gy was significantly associated with the risk of rubeosis $(p<0.001)$, the risk of NVG $(p=0.004)$, and subsequently the risk of visual acuity of less than $20 / 200$ $(\mathrm{p}=0.01)$.

\section{Discussion}

This study reports on ocular recurrence rate, survival rates, and toxicity for large non-peripapillary choroidal melanoma treated with proton therapy in Canada between 1995 and 2013. The optimal treatment method for large-sized melanoma remains a subject of debate.
We report a 5-year ocular recurrence-free rate of $85 \%$, which is lower than the rate of $90-97 \%$ reported in other studies on proton beam radiotherapy of medium-sized tumors $[3,6,15-17]$. Also, we found a 5 -year metastasisfree survival rate of $72 \%$ and an overall survival rate of $77 \%$, which is also lower than the rates of $80-90 \%$ reported in the literature $[3,6,15-17]$. However, our study cohort represented a challenging group compared to other studies due to several parameters. First of all, the median tumor diameter in our study was $13.6 \mathrm{~mm}$, and the median thickness (height) was $7.1 \mathrm{~mm}$. In other centers, where proton beam radiotherapy is used as a first choice for most patients with uveal melanomas $[5,15,16]$, the median thickness is typically lower as both small and large tumors are included. In contrast, centers that use proton beam radiotherapy more selectively, like us, when other eye-sparring methods are considered less likely to be successful, often include a higher number of large bulky tumors unsuitable for brachytherapy $[3,17]$. A retrospective study of 78 patients from the Loma Linda University Medical Center included patients with large-sized tumors in their evaluation of proton therapy and found a rate of metastasis-free survival $(76 \%)$, which is nearly identical to that in our study. A recently published multicenter study validated the AJCC staging system includ- 
ing more than 3,800 patients and found a 5-year metastasis-free survival rate of $85 \%$ in stage 2 (stage IIa + IIb) patients. Of our patients, $74 \%$ were classified as stage 2 according to the AJCC, and the 5-year metastasis-free survival was $81.8 \%$ (table 2 ).

Our patients were deemed unsuitable for brachytherapy, and therefore a direct comparison to the results of the COMS, which explored the efficacy of episcleral brachytherapy, cannot be made. The COMS only included medium-sized tumors defined as tumors with a maximum tumor height of $8 \mathrm{~mm}$ and a maximum basal diameter of $16 \mathrm{~mm}$. In our study, $48 \%$ of the patients had a tumor height above $8 \mathrm{~mm}$ or a basal diameter above 16 $\mathrm{mm}$ (COMS size large). Another important factor is that most of our tumors were located anterior to the equator, and $35 \%$ had involvement of the ciliary body, which is a well-known predictor of poor prognosis [16, 18-20].

Despite the unfavorable parameters, our study demonstrates acceptable outcomes for patients who were deemed unsuitable for other eye-sparing treatments.

The ocular recurrence rate was $15 \%$ after 5 years, and no ocular recurrence was seen after 5 years. Only 1 ocular recurrence was within the irradiated field. $\mathrm{T}$ stage alone was not a good predictor for ocular recurrence as half the recurrences were found in small $\mathrm{T} 1$ or $\mathrm{T} 2$ tumors with invasion into the ciliary body, whereas the other half had large tumors with a basal diameter above $16 \mathrm{~mm}$ (COMS size large). Instead, the 2010 AJCC anatomic staging [12], which takes into account both invasion into the ciliary body and extrascleral extension, was found to be better correlated with ocular recurrence and survival. An interesting finding was that all 7 ocular recurrences were found in tumors located anterior to the equator, and no recurrences were found in the 36 patients with a posterior tumor. Similarly, the survival rates were much better in patients with posteriorly located tumors. This was not only explained by the ciliary body involvement of the anteriorly located tumors but was probably also caused by a larger tumor size in the anterior group. Other authors have shown that posterior tumors involving the optic nerve or macula region carry a better prognosis than anteriorly located tumors, which may remain asymptomatic for a longer period of time, can be difficult to detect, and therefore often present in more advanced stages [19,21].

Out of 7 patients, 6 (86\%) with ocular recurrences developed liver metastasis and died. This rate is high compared to other studies [3, 6, 15-17], but again, it might be explained by the large tumor size in our cohort and the high fraction with ciliary body involvement. This underscores the importance of initial local control, and the question is whether patients with large anteriorly located tumors and/or invasion into the ciliary body should be enucleated upfront, or whether the metastatic risk is determined earlier in the disease course. One could speculate that these tumors may have an aggressive tumor biology (by histology or tumor genetics) at the time of diagnosis or that the high blood flow through the ciliary body necessary for muscle contraction and accommodation might induce a higher risk of dissemination. Unfortunately, biologic tumor features associated with aggressiveness such as chromosomal alterations and gene expression profiles could not be investigated in this study, as tumor tissue was not available in the early cohort patients $[19,20]$. It has recently been shown that analysis of the genetic status of the tumor together with the AJCC staging provided additional information on survival [22]. It is possible that high-risk patients could benefit from other treatment options or a combination of treatment modalities as for example systemic therapy including targeted agents and/or enucleation upfront, and this should be further investigated in future studies [23].

Finally, useful vision is important for patients, and in our study we found a gratifyingly high proportion of patients that maintained their vision after treatment. This could be explained by the fact that many were located anteriorly where a higher volume of the retina could be avoided, and we found that the risk of rubeosis, NVG, and blindness were highly associated with the volume of the retina getting more than $20 \mathrm{~Gy}$.

However, our study has limitations as it is a retrospective study with a limited number of patients, and therefore more detailed statistics could not be performed. Furthermore, patients were selected to this study based on a number of patient and tumor characteristics and ultimately the physician judgement.

In conclusion, proton therapy leads to acceptable survival and ocular recurrence rates in patients with large anteriorly located tumors, and radiation complications can be reduced by careful follow-up and vision maintained in a substantial proportion of patients.

\section{Acknowledgements}

We would like to thank the Danish Cancer Society (KB) and the Danish Society of Clinical Oncology (DSKO) for funding of travel and additional living expenses for Britta Weber in her postdoctoral fellowship at BC Cancer Agency. Special thanks to the Medical Physics Department at the BC Cancer Agency, and to the many support physicists at the TRIUMF cyclotron, Vancouver, B.C., Canada.
Weber/Paton/Ma/Pickles 


\section{Statement of Ethics}

The study was approved by the Research Ethics Board of the University of British Columbia.

\section{Disclosure Statement}

The authors have no conflict of interest to disclose.

\section{References}

1 Virgili G, Gatta G, Ciccolallo L, Capocaccia R, Biggeri A, Crocetti E, Lutz JM, Paci E: Incidence of uveal melanoma in Europe. Ophthalmology 2007;114:2309-2315.

-2 Char DH, Quivey JM, Castro JR, Kroll S, Phillips T: Helium ions versus iodine 125 brachytherapy in the management of uveal melanoma. A prospective, randomized, dynamically balanced trial. Ophthalmology 1993;100: $1547-1554$.

-3 Damato B, Kacperek A, Chopra M, Campbell IR, Errington RD: Proton beam radiotherapy of choroidal melanoma: the Liverpool-Clatterbridge experience. Int J Radiat Oncol Biol Phys 2005;62:1405-1411.

-4 Damato B, Kacperek A, Errington D, Heimann H: Proton beam radiotherapy of uveal melanoma. Saudi J Ophthalmol 2013;27:151157.

5 Gragoudas E, Li W, Goitein M, Lane AM, Munzenrider JE, Egan KM: Evidence-based estimates of outcome in patients irradiated for intraocular melanoma. Arch Ophthalmol 2002;120:1665-1671.

6 Jampol LM, Moy CS, Murray TG, Reynolds SM, Albert DM, Schachat AP, Diddie KR, Engstrom RE Jr, Finger PT, Hovland KR, Joffe L, Olsen KR, Wells CG: The COMS randomized trial of iodine 125 brachytherapy for choroidal melanoma. IV. Local treatment failure and enucleation in the first 5 years after brachytherapy. COMS report No. 19. Ophthalmology 2002;109:2197-2206.

-7 Muller K, Naus N, Nowak PJ, Schmitz PI, de Pan C, van Santen CA, Marijnissen JP, Paridaens DA, Levendag PC, Luyten GP: Fractionated stereotactic radiotherapy for uveal melanoma, late clinical results. Radiother Oncol 2012;102:219-224.
8 Muller K, Nowak PJ, de Pan C, Marijnissen JP, Paridaens DA, Levendag P, Luyten GP: Effectiveness of fractionated stereotactic radiotherapy for uveal melanoma. Int J Radiat Oncol Biol Phys 2005;63:116-122.

9 Macdonald EC, Cauchi P, Kemp EG: Proton beam therapy for the treatment of uveal melanoma in Scotland. Br J Ophthalmol 2011;95: 1691-1695.

10 Mosci C, Lanza FB, Barla A, Mosci S, Herault J, Anselmi L, Truini M: Comparison of clinical outcomes for patients with large choroidal melanoma after primary treatment with enucleation or proton beam radiotherapy. Ophthalmologica 2012;227:190-196.

11 Tran E, Ma R, Paton K, Blackmore E, Pickles $\mathrm{T}$ : Outcomes of proton radiation therapy for peripapillary choroidal melanoma at the $\mathrm{BC}$ Cancer Agency. Int J Radiat Oncol Biol Phys 2012;83:1425-1431.

12 Mellen PL, Morton SJ, Shields CL: American joint committee on cancer staging of uveal melanoma. Oman J Ophthalmol 2013;6:116118.

13 Goitein M, Miller T: Planning proton therapy of the eye. Med Phys 1983;10:275-283.

14 Wouters BG, Lam GK, Oelfke U, Gardey K, Durand RE, Skarsgard LD: Measurements of relative biological effectiveness of the $70 \mathrm{MeV}$ proton beam at TRIUMF using Chinese hamster V79 cells and the high-precision cell sorter assay. Radiat Res 1996;146:159-170.

$\checkmark 15$ Caujolle JP, Mammar H, Chamorey E, Pinon F, Herault J, Gastaud P: Proton beam radiotherapy for uveal melanomas at nice teaching hospital: 16 years' experience. Int J Radiat Oncol Biol Phys 2010;78:98-103.

16 Egger E, Schalenbourg A, Zografos L, Bercher L, Boehringer T, Chamot L, Goitein G: Maximizing local tumor control and survival after proton beam radiotherapy of uveal melanoma. Int J Radiat Oncol Biol Phys 2001;51:138147.
17 Dendale R, Lumbroso-Le Rouic L, Noel G Feuvret L, Levy C, Delacroix S, Meyer A, Nauraye $\mathrm{C}$, Mazal A, Mammar $\mathrm{H}$, Garcia $\mathrm{P}$, D’Hermies F, Frau E, Plancher C, Asselain B, Schlienger P, Mazeron JJ, Desjardins L: Proton beam radiotherapy for uveal melanoma: results of Curie Institut-Orsay proton therapy center (ICPO). Int J Radiat Oncol Biol Phys 2006;65:780-787.

18 Damato BE, Heimann H, Kalirai H, Coupland SE: Age, survival predictors, and metastatic death in patients with choroidal melanoma: tentative evidence of a therapeutic effect on survival. JAMA Ophthalmol 2014;132:605613

19 Li W, Gragoudas ES, Egan KM: Metastatic melanoma death rates by anatomic site after proton beam irradiation for uveal melanoma. Arch Ophthalmol 2000;118:1066-1070.

20 Shammas HF, Blodi FC: Prognostic factors in choroidal and ciliary body melanomas. Arch Ophthalmol 1977;95:63-69.

-21 Seddon JM, Albert DM, Lavin PT, Robinson $\mathrm{N}$ : A prognostic factor study of disease-free interval and survival following enucleation for uveal melanoma. Arch Ophthalmol 1983; 101:1894-1899.

22 Bagger M, Andersen MT, Andersen KK, Heegaard S, Andersen MK, Kiilgaard JF: The prognostic effect of American Joint Committee on Cancer staging and genetic status in patients with choroidal and ciliary body melanoma. Invest Ophthalmol Vis Sci 2014;56: 438-444.

23 Field MG, Harbour JW: Recent developments in prognostic and predictive testing in uveal melanoma. Curr Opin Ophthalmol 2014;25: 234-239. 